\title{
Adding the head-tilt-chin-lift technique to adult compression-only CPR by untrained bystanders
}

\author{
Anthony M.-H. Ho MD, Song Wan MD, David C. Chung MD
}

$\mathrm{T}$ he global incidence of out-of-hospital cardiac arrest is $213.1(\mathrm{SD} \pm 177)$ per 100000 population. ${ }^{1}$ More than 20000 people have out-of-hospital cardiac arrest each year in Canada, where cardiopulmonary resuscitation (CPR) rates rarely exceed $25 \% .^{2}$ The latest (2010) guidelines from the International Liaison Committee on Resuscitation recommend chest-compression-only CPR for untrained bystanders attending to adults with outof-hospital cardiac arrest (level 1 evidence). ${ }^{1}$ The conventional approach of chest compression plus rescue breathing (the latter consists of the head-tilt-chin-lift technique plus mouth-tomouth ventilation) continues to be recommended for pediatric and adult CPR performed by trained bystanders and rescuers. ${ }^{1}$

The elimination of rescue breathing from the CPR protocol for untrained bystanders was based on several compelling physiologic and pragmatic considerations. Mouth-to-mouth ventilation delays the initiation of and interrupts chest compressions; positive-pressure ventilation decreases venous return; hyperventilation may occur, resulting in cerebral and coronary vasoconstriction; lay people may be reluctant to perform CPR if it involves intimate contact; ventilation interspersed with chest compressions is taxing; rescue breaths may insufflate the stomach and interfere with gasping; and the primary cause of out-of-hospital cardiac arrest in adults is seldom respiratory in nature. However, wholesale elimination of ventilation from the adult CPR protocol for untrained lay people may be misguided.

The decision to eliminate rescue breathing from the adult CPR protocol for untrained bystanders was supported by a meta-analysis ${ }^{3}$ of three randomized controlled trials (RCTs). This meta-analysis showed that chest-compressiononly CPR was associated with an improved chance of survival compared with conventional CPR (compression:ventilation ratio 15:2) (14\% [211/1500] v. $12 \%$ [178/1531]; risk ratio 1.22 , 95\% confidence interval [CI] 1.01-1.46; absolute increase in survival $2.4 \%$, 95\% CI $0.1-4.9$; number needed to treat $41,95 \%$ CI $20-1250$ ). The individual RCTs were insufficiently powered to draw such strong conclusions. ${ }^{3}$ All three RCTs used a similar methodology: once it was ascertained that the situation warranted inclusion in the study, an untrained bystander was instructed over the phone to perform either conventional CPR or chest-compression-only CPR. However, because conventional CPR would have taken longer to teach, the RCTs actually compared CPR with a high compression fraction (started sooner) and CPR with a low compression fraction (delayed start).

An analysis of observational studies reporting data on the technique of bystander resuscitation, collected by paramedics at the scene, showed that compression-only CPR was noninferior ${ }^{3}$ or superior ${ }^{4}$ to conventional CPR. However, most of these observational studies were conducted in urban centres, where paramedic response time and time to hospital are short. ${ }^{5}$ In rural areas, where response times are longer and defibrillation cannot be given immediately, survival after CPR that excludes ventilation for a prolonged period is substantially less likely. An observational study in Japan published in 2011 on out-of-hospital cardiac arrest found that conventional CPR was associated with better outcomes than chest-compression-only CPR for one-month survival (adjusted odds ratio [OR] $1.17,95 \%$ CI 1.06-1.29) and neurologically favourable one-month survival (adjusted OR $1.17,95 \%$ CI 1.01-1.35); better outcomes were seen among younger patients with events of noncardiac origin and among patients ex-

\section{- Ker Points}

- Recent guidelines recommend chest-compression-only cardiopulmonary resuscitation (CPR) by untrained bystanders in adults with out-ofhospital cardiac arrest.

- Rescue breathing (consisting of the head-tilt-chin-lift technique plus mouth-to-mouth ventilation) was eliminated from the guidelines to make CPR easier for untrained lay people.

- The evidence supporting the elimination of rescue breathing in the guidelines is open to challenge.

- Physiologic evidence supports the addition of head tilt plus chin lift or chin lift to chest-compression-only CPR by untrained bystanders in adults with out-of-hospital cardiac arrest. 
periencing a delay of up to 10 minutes before the start of CPR after the event was witnessed for noncardiac events and also for all events combined. ${ }^{6}$ Some animal studies in support of compression-only CPR had ignored basic anatomic differences between animals and humans; for example, pigs and dogs have straight airways that do not obstruct in the comatose state.

The elimination of rescue breathing from the adult CPR protocol is especially dangerous for patients with cardiac arrest from a noncardiac cause: in one study, $15 \%$ of the cardiac arrests had noncardiac causes, and over half of these were primarily respiratory. ${ }^{7}$ In an observational study involving children who had out-of-hospital cardiac arrest, patients whose cardiac arrest was from a respiratory cause had better survival with conventional CPR than with chest-compressiononly CPR. ${ }^{8}$

The aforementioned issues identified by the International Liaison Committee on Resuscitation apply to mouth-to-mouth, positive-pressure ventilation. However, rescue breathing is a twopart intervention: head tilt plus chin lift, and delivery of rescue breaths. The head-tilt-chin-lift technique, the key to overcoming obstruction in the upper airway in unconscious patients, ${ }^{9}$ is not the reason for all the undesirable effects of rescue mouth-to-mouth breathing. It is easy to perform $^{9}$ if another rescuer is present, and no intimate contact is necessary. The manoeuvre does not affect venous return or interfere with gasping. Any passive ventilation will deliver air; rescue breaths deliver $16 \%$ oxygen and $4 \%$ carbon dioxide. Furthermore, application of the headtilt-chin-lift technique by a second rescuer allows uninterrupted chest compressions to follow. Ventilation is crucial when the arrest has a respiratory cause.

Ventilation should ideally start within minutes after the arrest. Without the head-tilt-chinlift technique, any passive ventilation during chest compression may only generate tidal volumes smaller than anatomic dead space. ${ }^{10}$ In a study comparing the effectiveness of techniques for opening an airway obstructed by the tongue, the head-tilt-chin-lift technique produced adequate airway patency in $90.8 \%$ of patients who were anesthetized and given mouth-to-mouth ventilation. In 30 spontaneously breathing patients who were anesthetized and administered chin lift, tidal volumes of 50-250 mL, 250$400 \mathrm{~mL}$ and $>400 \mathrm{~mL}$ were achieved in 2,7 and 21 patients, respectively. ${ }^{9}$

Anesthesiologists typically maintain airway patency in spontaneously breathing or artificially ventilated patients who are anesthetized using chin lift with some head tilt. The small percentage of patients whose airway remains obstructed despite chin lift with or without head tilt can almost always be managed using an oropharyngeal airway. Passive ventilation through an oropharyngeal airway results in substantially higher tidal volumes and, among patients with witnessed ventricular fibrillation, a survival advantage over conventional CPR. ${ }^{11}$ Although the head-tilt-chin-lift technique does not provide the same level of patency as an oropharyngeal airway, airway patency clearly benefits passive ventilation and may influence survival.

Would the widespread use of the head-tiltchin-lift technique in CPR increase the risk of exacerbating injuries to the cervical spine? Cervical instability affects less than $4 \%$ of patients who have serious blunt trauma, a group with notoriously poor outcomes when cardiac arrest occurs. The American College of Emergency Physicians recommends chin lift (plus, if necessary, limited head tilt) for airway patency even in an injured patient. ${ }^{12}$

The head-tilt-chin-lift technique is easier to learn and to execute in a crisis than full rescue breathing. The physiologic argument and evidence ${ }^{11,13}$ supporting the addition of this technique to compression-only CPR by untrained bystanders in adults with out-of-hospital cardiac arrest are compelling. Uninterrupted chest compressions with passive ventilation through the use of head tilt plus chin lift or chin lift alone is applicable across all age groups, presentations, causes and settings (i.e., rural or urban and witnessed or nonwitnessed). With an average survival rate of $14 \%$ with compression-only CPR, ${ }^{3}$ there is a lot of room for improvement. Studies of chest-compression-only CPR that incorporates the head-tilt-chin-lift technique for two-person CPR by untrained bystanders following out-of-hospital cardiac arrest in adults are warranted and should be given top priority.

See references, www.cmaj.ca/lookup/suppl/doi:10.1503 /cmaj.131847/-/DC1

Affiliations: Department of Anesthesiology and Perioperative Medicine (Ho), Queen's University, Kingston General Hospital, Kingston, Ont.; Department of Surgery (Wan) and Department of Anaesthesia and Intensive Care (Chung), The Chinese University of Hong Kong, Hong Kong

Contributors: All of the authors contributed substantially to the preparation of this manuscript, have approved the final version submitted for publication and have agreed to act as guarantors of the work.

Acknowledgement: This work has been supported through departmental and institutional resources. 